\title{
The high-affinity HSP90-CHIP complex recognizes and selectively degrades phosphorylated tau client proteins
}

\author{
Chad A. Dickey, ${ }^{1}$ Adeela Kamal, ${ }^{2}$ Karen Lundgren, ${ }^{2}$ Natalia Klosak, ${ }^{1}$ Rachel M. Bailey, ${ }^{1}$ \\ Judith Dunmore, ${ }^{1}$ Peter Ash, ${ }^{1}$ Sareh Shoraka, ${ }^{1}$ Jelena Zlatkovic, ${ }^{1}$ Christopher B. Eckman, ${ }^{1}$ \\ Cam Patterson, ${ }^{3}$ Dennis W. Dickson, ${ }^{1}$ N. Stanley Nahman Jr., ${ }^{4}$ Michael Hutton, ${ }^{1}$ \\ Francis Burrows, ${ }^{2}$ and Leonard Petrucelli ${ }^{1}$
}

\begin{abstract}
${ }^{1}$ Department of Neuroscience, Mayo Clinic College of Medicine, Jacksonville, Florida, USA. ${ }^{2 B i o g e n ~ I d e c ~ R e s e a r c h, ~ C o m m e r c i a l ~ C a p a b i l i t i e s, ~}$ San Diego, California, USA. ${ }^{3}$ School of Medicine, University of North Carolina at Chapel Hill, Chapel Hill, North Carolina, USA. ${ }^{4}$ College of Medicine, University of Florida, Gainesville, Florida, USA.
\end{abstract}

\begin{abstract}
A primary pathologic component of Alzheimer's disease $(\mathrm{AD})$ is the formation of neurofibrillary tangles composed of hyperphosphorylated tau (p-tau). Expediting the removal of these p-tau species may be a relevant therapeutic strategy. Here we report that inhibition of Hsp90 led to decreases in p-tau levels independent of heat shock factor 1 (HSF1) activation. A critical mediator of this mechanism was carboxy terminus of Hsp70-interacting protein (CHIP), a tau ubiquitin ligase. Cochaperones were also involved in Hsp90-mediated removal of p-tau, while those of the mature Hsp90 refolding complex prevented this effect. This is the first demonstration to our knowledge that blockade of the refolding pathway promotes p-tau turnover through degradation. We also show that peripheral administration of a novel Hsp90 inhibitor promoted selective decreases in p-tau species in a mouse model of tauopathy, further suggesting a central role for the $\mathrm{Hsp} 90$ complex in the pathogenesis of tauopathies. When taken in the context of known high-affinity $\mathrm{Hsp} 90$ complexes in affected regions of the $\mathrm{AD}$ brain, these data implicate a central role for $\mathrm{Hsp} 90$ in the development of $\mathrm{AD}$ and other tauopathies and may provide a rationale for the development of novel Hsp90-based therapeutic strategies.
\end{abstract}

\section{Introduction}

Intracellular aggregation of abnormal species of phosphorylated tau (p-tau), the microtubule-associated protein, is a major pathologic feature of a family of neurodegenerative disorders collectively referred to as the tauopathies $(1,2)$. The most common tauopathy is Alzheimer's disease (AD), in which p-tau aggregates in neurofibrillary tangles, in dystrophic neurites in senile plaques, and in cell processes in the neuropil $(3,4)$. While these lesions represent visible evidence of p-tau aggregation, the formation of soluble toxic tau species may be more important mediators of tau-associated neurodegeneration. If so, then decreasing $\mathrm{p}$-tau levels through refolding or degradation may be a plausible therapeutic strategy.

Aberrant neuronal protein aggregation in the tauopathies may result in part from impaired chaperone-mediated protein ubiquitination and degradation $(5,6)$. Hsps have been shown to be upregulated in AD brain (7-9). Molecular chaperones are capable of reducing p-tau concentrations and have been shown to prevent tau-associated cellular toxicity $(10,11)$. Further support for the role of the cytosolic chaperone network in the processing of tau proteins is the demonstration that deletion of the Hsp70 cochap-

Nonstandard abbreviations used: $\mathrm{AD}$, Alzheimer's disease; biotin-GA, biotinlabeled GA; CHIP, carboxy terminus of Hsp70-interacting protein; GA, geldanamycin; GSK3 $\beta$, glycogen synthase kinase $3 \beta$; Hsc, heat shock cognate; Hop, Hsp70/Hsp90organizing protein; HSF1, heat shock factor 1; Htau mouse, transgenic mouse humanized for the tau gene; MARK2, microtubule-affinity regulating kinase 2; myc-CHIP, myc-tagged CHIP; PAR-1, PAR-1 serine/threonine kinase; p-tau, phosphorylated tau; V5-tau, V5-tagged tau.

Conflict of interest: The authors have declared that no conflict of interest exists. Citation for this article: J. Clin. Invest. 117:648-658 (2007). doi:10.1172/JCI29715. erone carboxy terminus of Hsp70-interacting protein (CHIP) results in accumulation of soluble p-tau in the brain (12).

Hsp90 is a molecular chaperone that is involved in the folding and stabilization of many client proteins. The naturally occurring ansamycin antibiotic geldanamycin (GA) inhibits Hsp90 chaperone function by reducing ATPase activity (13). This has 2 functional consequences: (a) enhanced degradation of client proteins bound by Hsp90 and (b) activation of heat shock factor 1 (HSF1), a transcriptional activator of other stress-induced chaperone proteins (14). Tumor cells possess Hsp90/chaperone complexes that exhibit high affinity for Hsp90 inhibitors (15), providing a rationale for current clinical trials of Hsp90 inhibitors in the treatment of cancer.

We have previously identified several Hsp90 inhibitors with suitable pharmacokinetic profiles for potential therapeutic use in neurodegenerative disease (16). These compounds promoted selective proteasome-dependent degradation of aberrant $\mathrm{p}$-tau species in vitro. In the present study, we assessed the effect of the Hsp90 inhibitor EC102 in facilitating the degradation of aberrant p-tau species using a humanized tau transgenic mouse. Our results demonstrated that the peripheral administration of EC102 promoted selective degradation of $\mathrm{p}$-tau species in the brains of these animals. The results support the hypothesis that Hsp90 is involved in the pathogenesis of $\mathrm{AD}$ and associated tauopathies and suggest that the Hsp90 complex is a practical target for the treatment of neurodegenerative tauopathies.

\section{Results}

EC102 is a blood-brain barrier-permeable Hsp90 inbibitor. We previously identified several low-molecular weight Hsp90 inhibitors that 


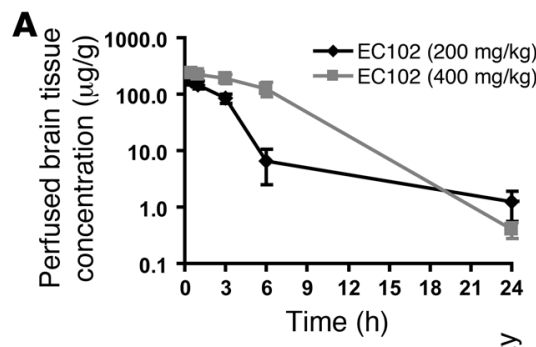

C

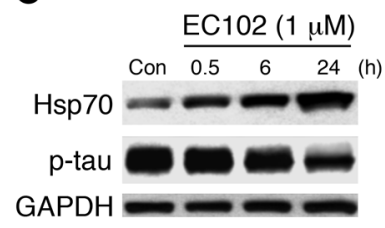

B

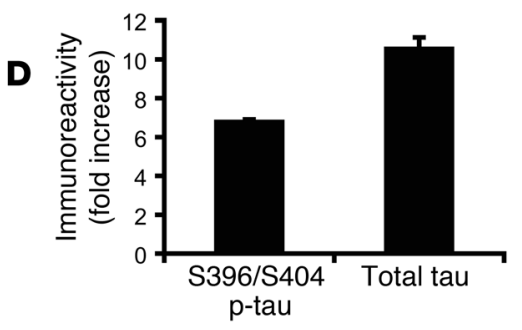

Figure 1

EC102 crosses the blood-brain barrier and reduces tau levels in cells after 24 hours. (A) CD-1 mice were injected i.p. with the indicated doses of EC102 and harvested 1, 3, 6, and 24 hours after injection. Brain levels of EC102 were assessed by HPLC analysis. Greater than $50 \%$ concentration was maintained for 3 hours with $200 \mathrm{mg} / \mathrm{kg}$ without detectable toxicity. (B) CD-1 mice were injected i.p. with $200 \mathrm{mg} / \mathrm{kg} \mathrm{EC102}$ or equivalent vehicle control (Con) to demonstrate the latency in Hsp70 induction following Hsp90 inhibition. After 6 hours, a slight increase in Hsp70 levels was observed in EC102-treated brain tissue, followed by a robust induction at 24 hours compared with vehicle-treated brain tissue. (C) HeLa cells overexpressing V5-tau were treated with a 1- $\mu \mathrm{M}$ concentration of EC102 for the indicated time points. p-tau, Hsp70, and GAPDH levels were assessed by Western blot. p-tau levels were modestly decreased 6 hours after treatment, with maximal reduction seen at 24 hours after treatment. Hsp70 levels were increased in a time-dependent manner. (D) In-cell Western analysis showed that $60 \%-65 \%$ of total tau was converted to $p$-tau in HeLa cells 24 hours after transfection with V5-tau.

preferentially degrade p-tau species in cells overexpressing P301L mutant tau $(16,17)$. Results of subsequent studies indicated that EC102 was the most effective agent. The drug crossed the bloodbrain barrier following i.p. administration in mice and attained a brain $\mathrm{IC}_{50}$ for 3 hours at a concentration of $200 \mathrm{mg} / \mathrm{kg}$ (Figure 1A).
In addition, Hsp70 levels were increased in the brains of treated mice after 6 hours (Figure 1B). Thus, for all subsequent studies, EC102 was used to inhibit Hsp90 activity.

In order to determine the optimal interval for in vitro efficacy, HeLa cells overexpressing V5-tagged tau (V5-tau) were treated with a $1-\mu \mathrm{M}$ dose of EC102 and harvested at different time points. Figure $1 \mathrm{C}$ shows that 24 hours was the optimal time point, based on robust Hsp induction and $\mathrm{p}$-tau reduction. The reduction in $\mathrm{p}$-tau could not be attributed to reduced mRNA expression, because the V5-tau construct used was driven by a heterologous CMV promoter, the same promoter used to drive $\alpha$-synuclein expression in these cells, which was unaffected by Hsp90 inhibitor treatment (Supplemental Figure 1; supplemental material available online with this article; doi:10.1172/JCI29715DS1).

In HeLa cells overexpressing V5-tau, p-tau was quantified using an in-cell Western assay $(16,18)$. Approximately $65 \%$ of the total tau was phosphorylated at S396/ S404 (Figure 1D), a p-tau epitope that is preferentially degraded by Hsp90 inhibition (16).

The processing pathway of client proteins of the chaperone network is shown in Figure 2: Hsp40 and Hsp70 form the initial recognition complex to prevent the aggregation of aberrant proteins. CHIP is likely involved early in the cycle because of its interaction with Hsp70 (19). Hsp70 client proteins may then be transferred to the Hsp90 complex, a process dependent upon Hsp70/Hsp90-organizing protein (Hop; refs. 20-23). Subsequently, the Hsp90 complex can be refolded and dephosphorylated by binding with cochaperones (such as P23 and Pin1, respectively; refs. 24,25 ) or degraded via the ubiquitin-proteasome system. In the latter pathway, Hsp90 is degraded. Hsp90 also suppresses HSF1 activity by binding the factor. Following Hsp90 degradation, free HSF1 translocates to the nucleus and initiates transcription of other Hsps. Chemical inhibition of Hsp90 promotes both of the above functions. Using siRNAs to target several of the above mediators of the chaperone network,

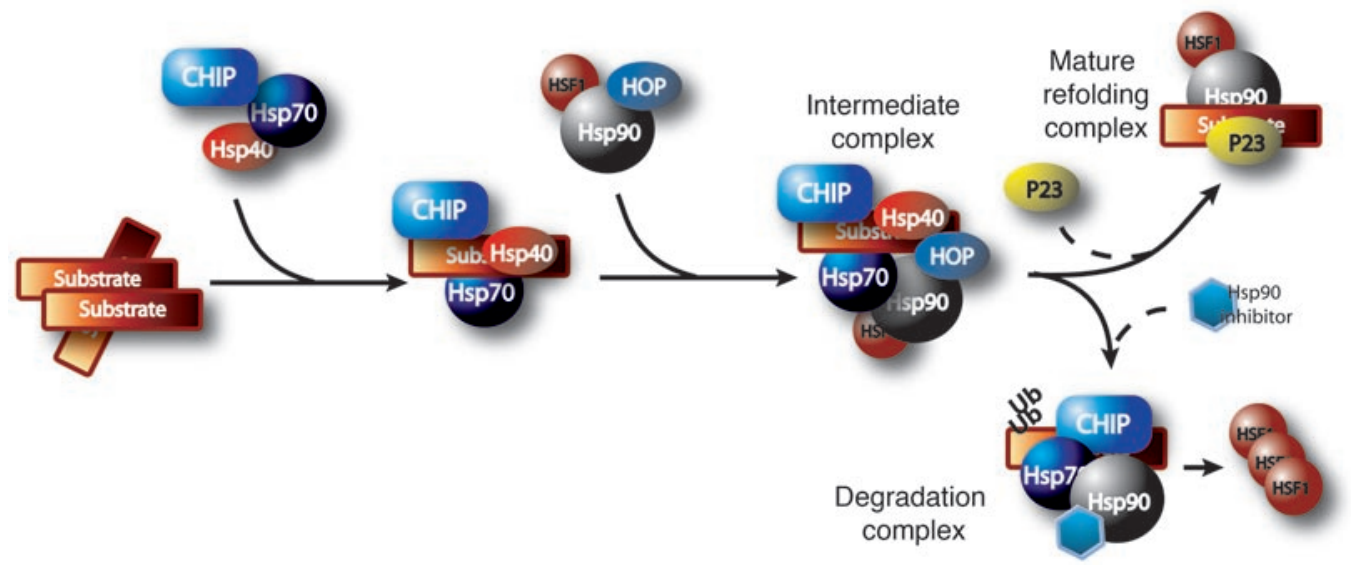

Figure 2

Proposed mechanism of chaperone/client pathway. A substrate may initially be recognized by the Hsp40/Hsp70 complex with $\mathrm{CHIP}$ as the cochaperone/E3 ubiquitin ( $\mathrm{Ub}$ ) ligase. Transfer of the substrate to the Hsp90 complex is facilitated by Hop. There remain 2 fates for the substrate: dephosphorylation and refolding or ubiquitin-dependent proteasomal degradation. The mechanisms dictating which pathway is taken remain undefined. Hsp90 expression inhibits HSF1 activity by direct binding, which prevents HSF1 phosphorylation and trimerization. When Hsp90 is inhibited, Hsp90 levels are decreased, releasing HSF1, which in turn promotes de novo transcription of Hsps. 


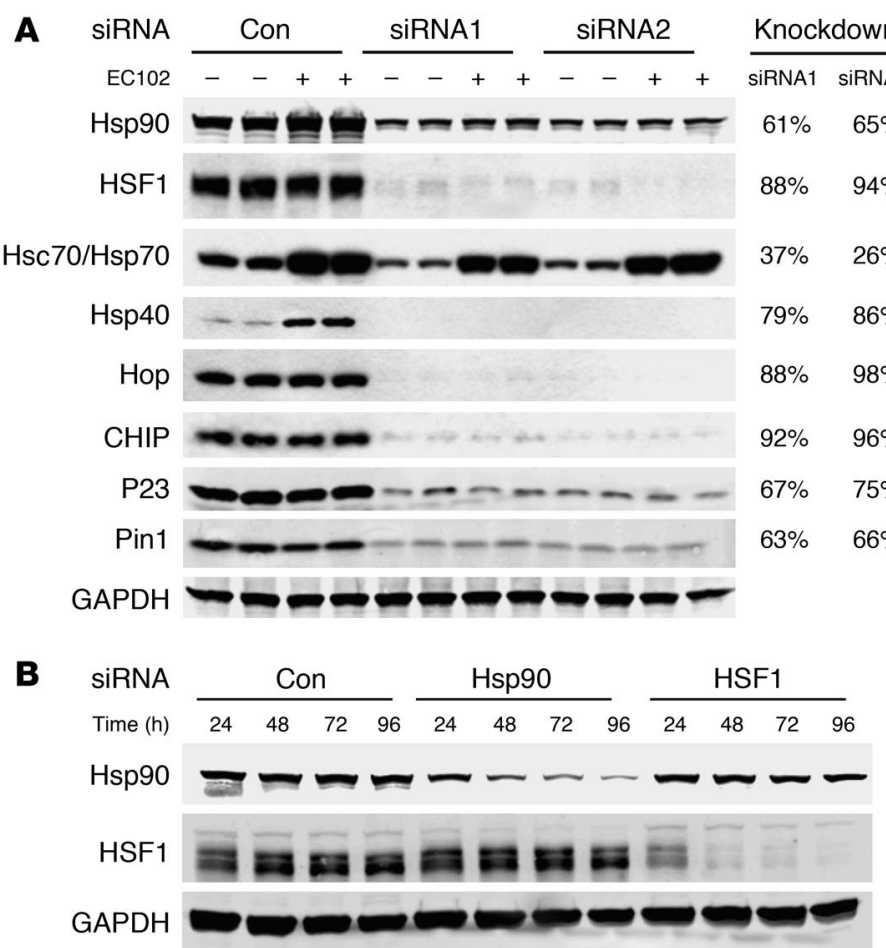

Figure 3

Targeted knockdown of primary components of the chaperone pathway promotes robust and rapid reductions in protein levels. (A) HeLa cells were transfected in duplicate with a scrambled nonsilencing siRNA control or 2 independent siRNAs per gene, targeting the indicated components of the chaperone cycle. After 72 hours, the cells were treated with EC102 $(1 \mu \mathrm{M})$ for an additional 24 hours and harvested for Western analyses. Knockdown efficiency at the protein level for both siRNAs targeting 7 of the 8 genes was greater than $60 \%$ and averaged $80 \%$. Only siRNAs targeting $\mathrm{Hsp} 70$ had modest reductions of approximately $30 \%$. Induction of Hsps by EC102 was also prevented by their knockdown, with the exception of Hsp70. GAPDH immunoreactivity was assessed on each membrane to control for loading differences. The panel shown is representative for all membranes. Based on similar knockdown efficiencies, these siRNAs were pooled for subsequent studies. Quantitation was assessed by densitometry. (B) HeLa cells were transfected with either nonsilencing siRNA control or 2 siRNA pools targeting Hsp90 or HSF1. The cells were harvested at the indicated time points. Partial knockdown was apparent after 24 hours for both genes, and immunoreactivity decreased further with each additional 24-hour interval. The 72-hour time point was chosen for subsequent studies to avoid issues associated with toxicity. we attempted to elucidate whether a similar mechanism could be applied to the degradation of p-tau.

Two siRNAs (QIAGEN) per target gene were characterized for knockdown potency in the presence and absence of EC102. Figure 3 A shows that both agents demonstrated similar knockdown efficiency regardless of EC102 exposure. These siRNAs were pooled in all subsequent studies, consistent with the work of others $(26,27)$. In order to determine the most appropriate time to administer EC102 to HeLa cells expressing V5-tau, we assessed the inhibitory time course of the above siRNAs for Hsp90 and HSF1 expression. Protein suppression was apparent as early as 24 hours, but was optimal at 72 and 96 hours for both inhibitory RNAs (Figure 3B). On this basis, the 72-hour time point was used in all subsequent studies.

The constitutive chaperone complex mediates tau degradation by $\mathrm{Hsp} 90$ inbibition. In order to investigate the mechanism by which Hsp90 inhibition reduced p-tau expression, EC102 was used to assess HSF1 and Hsp90 expression in HeLa cells overexpressing tau. In cells transfected with nonsilencing siRNA, EC102 reduced p-tau levels by $35 \%-40 \%$. In contrast, siRNA suppression of constitutive cytosolic Hsp90 expression completely abrogated EC102-
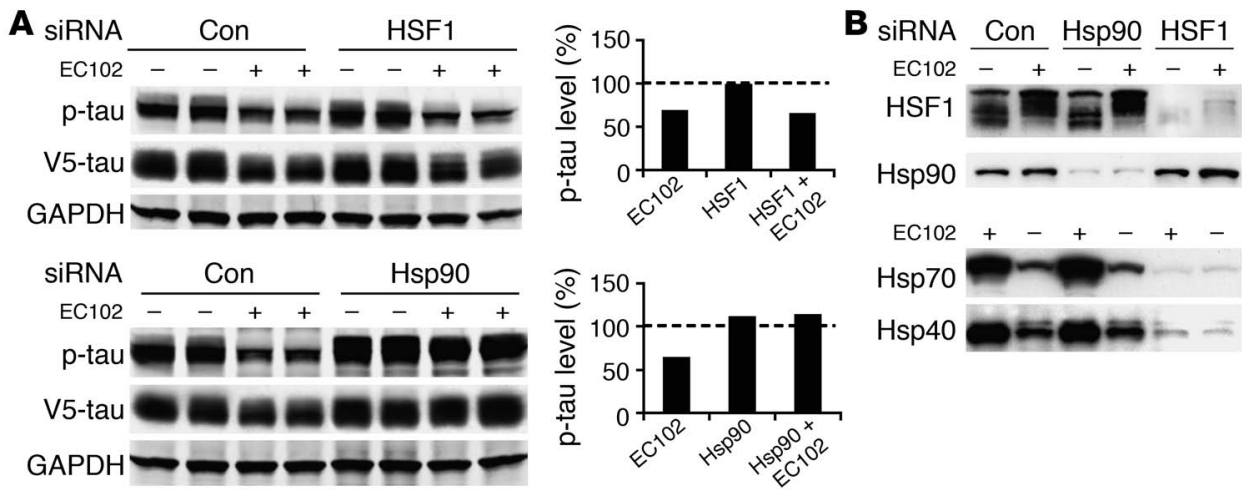

Figure 4

Reductions in p-tau by Hsp90 inhibition are primarily mediated by a constitutive, not an inducible, chaperone response. (A) HeLa cells were transfected in duplicate with nonsilencing control, Hsp90, or HSF1 siRNA pools and incubated for 72 hours. The cells were then transfected with V5-tau and harvested after 24 hours' EC102 exposure. EC102 caused robust reductions in p-tau and V5 immunoreactivity in cells transfected with a nonsilencing control or HSF1 siRNA (approximately 35\%), while Hsp90 knockdown prevented this reduction. Densitometric values for Hsp90 and HSF1 siRNA pools are represented in separate graphs as a percentage of the optical density for nontransfected, vehicle-treated control cells after GAPDH normalization (dashed line). (B) HSF1 knockdown prevented Hsp40 and Hsp70 induction by EC102, while Hsp90 knockdown had no effect on either of these Hsps. Hsp90 levels were unaffected by HSF1 knockdown. EC102 treatment caused a shift in the distribution of HSF1 species, suggestive of phosphodependent activation. 
A

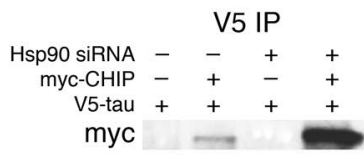

Inputs

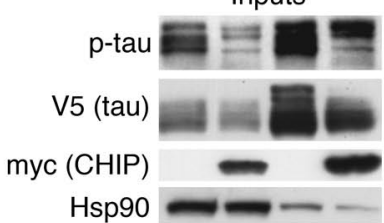

B

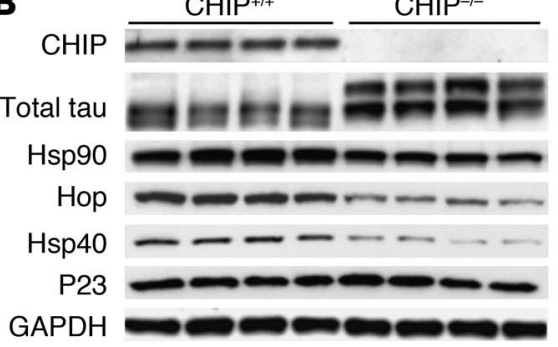

C

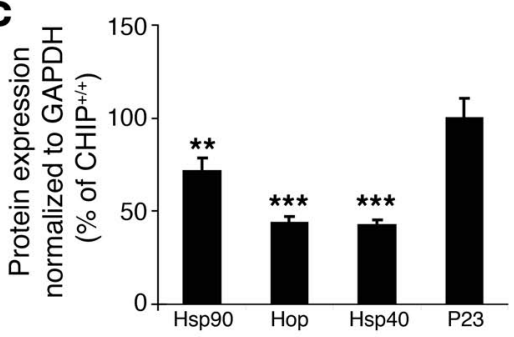

mediated tau reductions (Figure 4A). Despite incomplete Hsp90 knockdown, its suppression still attenuated EC102-mediated p-tau degradation (Figure 4A). Suppression of HSF1 expression by RNA interference prevented upregulation of both Hsp 40 and Hsp70 (Figure 4B), yet had no effect on EC102-mediated p-tau reductions. In control studies, the same expression cassette had no effect on $\alpha$-synuclein expression (Supplemental Figure 1). Taken together, these data suggest that p-tau was processed via the constitutive Hsp90 refolding system rather than being dependent on de novo transcription of Hsp chaperones stimulated by HSF1.

Cochaperones involved in the refolding and dephosphorylation of aberrant proteins initially prevent degradation. In order to assess the role of other components of the chaperone network in the degradation of p-tau, siRNAs were used to knock down constitutive expression of CHIP, heat shock cognate 79 (Hsc79)/Hsp70, Hsp40, and Hop. Figure $5 \mathrm{~A}$ shows that specific RNA interference for each of these proteins blocked EC102-mediated p-tau degradation, further suggesting a role for the cognate chaperone system in preventing p-tau degradation (quantification of these changes are presented in Figure 5B as a percentage of nontransfected, vehicle-treated cells). In control studies, drug efficacy was substantiated by elevated Hsp70 levels (Supplemental Figure 2) and reduced p-tau levels in cells receiving nonsilencing siRNA. In addition, elevation in Hsp70 expression was not altered by suppression of these chaperones (excluding siRNA directly targeting Hsc70/Hsp70, Figure $3 \mathrm{~A}$, and HSF1, Supplemental Figure 2). Finally, suppression of the inducible chaperone Hsp27 had no effect on Hsp90 inhibitor-mediated degradation of p-tau (data not shown) despite its association with tau in $\mathrm{AD}$ brain (10).

\section{Figure 6}

The unique cochaperone CHIP is essential for Hsp90 inhibitor-mediated tau degradation and regulates the levels of other chaperones as well as Hsp90. (A) HeLa cells transfected with Hsp90 siRNA were subsequently transfected with V5-tau with or without myc-CHIP. Tau accumulated when Hsp90 expression was reduced; however, this accumulation was abrogated by CHIP and the amount of coimmunoprecipitated tau/CHIP complexes increased in the absence of Hsp90. (B) Chaperone protein levels were assessed in $\mathrm{CHIP}^{-1-}$ brain tissue by Western blot analysis. The absence of CHIP and elevation in total tau levels were confirmed. Both Hsp40 and Hsp90 levels were decreased. In addition, the non-HSF1-mediated cochaperone, Hop, was also significantly decreased in $\mathrm{CHIP}^{-/-}$mice. P23 levels remained unchanged compared to GAPDH levels. (C) Quantification was assessed by standard densitometry. Error bars represent SD of the $4 \mathrm{CHIP}^{-/-}$mice. ${ }^{* *} P<0.01 ;{ }^{* * *} P<0.001$ versus $\mathrm{CHIP}^{+/+}$.

Suppression of the cochaperones P23 and Pin1 functionally mimicked EC102 activity by causing significant reductions in p-tau levels. Figure 5C shows that P23 siRNA reduced p-tau levels to an extent similar to that of EC102, and Pin1 knockdown reduced p-tau levels to an even greater extent than did EC102. Figure 5D shows the effect of each siRNA on total V5 immunoreactivity. In these experiments, suppression of Hsp90, CHIP, Hop, and Hsp40 caused total tau levels to increase by approximately $40 \%$. Neither HSF1 nor Hsp 70 affected tau levels. P23 and Pin1 reduced total tau levels by $32 \%$ and $76 \%$, respectively. The implications of these results, shown in Figure 5E, suggest that damaged client proteins (i.e., p-tau) are preferentially repaired through restorative chaperone activity, rather than by protein degradation pathways.

CHIP is a critical regulator of the chaperone response and Hsp90 inhibitor-mediated $p$-tan degradation. We previously demonstrated that CHIP is a central component for the degradation of $\mathrm{p}$-tau in vivo (16). CHIP is also a functional binding partner of Hsp70 and Hsp90 $(11,28)$. We therefore wanted to better understand both the role that CHIP was playing in Hsp90 inhibitor-mediated p-tau degradation and the interactions between this ubiquitin ligase and other chaperone components.

In order to assess the role of CHIP in Hsp90 inhibitor-mediated p-tau degradation, HeLa cells were treated with Hsp90 siRNA and transfected with V5-tau in the presence or absence of myc-tagged CHIP (myc-CHIP). Tau accumulated when Hsp90 expression was blocked. In the presence of CHIP overexpression, tau accumulation was prevented. In the presence of Hsp90 inhibition, V5-tau from cells overexpressing CHIP was coimmunoprecipitated, revealing that the number of CHIP/p-tau complexes was increased. These data indicate that CHIP can complex with tau, despite reductions in Hsp90 levels (Figure 6A).

In order to assess the interactions between CHIP and other chaperone proteins, we assessed the levels of several other members of the chaperone network in brain homogenates from $\mathrm{CHIP}^{-/-}$mice. Using this model, we have previously shown that $\mathrm{CHIP}^{-/-}$mice have increased p-tau (14). In the present work, Figure 6, B and C, demonstrate that these mice showed significantly reduced levels of Hop, Hsp90, and Hsp40. Levels of the cochaperone P23 were unchanged.

CHIP fails to recognize tan phosphorylated at normal serine residues; evidence for selective degradation. The above data suggest that p-tau can be targeted for degradation by inhibition of Hsp90. On the other hand, we previously reported that tau phosphorylated by microtubule-affinity regulating kinase 2/PAR-1 serine/threonine kinase 


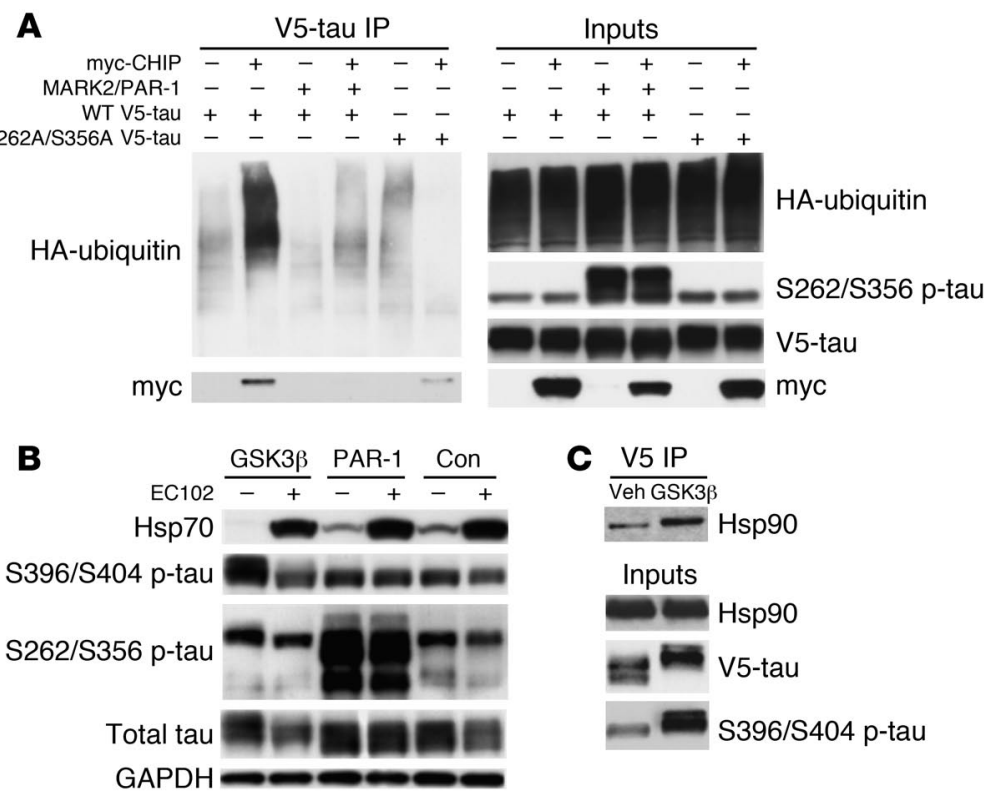

\section{Figure 7}

Phosphorylation at S262/S356 prevents ubiquitination and degradation of tau by either CHIP or Hsp90 inhibition, but does not abolish $\mathrm{CHIP} / \mathrm{Hsp} 90$ binding, further implicating ubiquitination of $\mathrm{p}$-tau by CHIP as a necessary component for Hsp90mediated degradation. (A) HEK293 cells transfected with either wild-type V5-tau or V5-tau harboring the double mutation of S262A/S356A were cotransfected with myc-CHIP, PAR-1, or both. V5 coimmunoprecipitation showed that CHIP bound to and greatly enhanced the polyubiquitination of wild-type tau; however, PAR-1 phosphorylation of tau prevented this interaction. Mutation of the S262 and S356 sites to alanine residues attenuated CHIP binding; however, ubiquitination activity was entirely abrogated. Inputs confirmed the hyperphosphorylation of wild-type tau at the S262/S356 residues in the presence of PAR-1. (B) HeLa cells expressing wild-type V5-tau were cotransfected with constitutively active GSK3 $\beta$, PAR-1, or empty vector and then treated with EC102 or vehicle. In the absence of EC102, constutively active GSK3 $\beta$ promoted the phosphorylation of tau at S396/S404 relative to empty vector and PAR-1 promoted the phosphorylation of tau at S262/S356 relative to empty vector. In the presence of EC102, phosphorylated S396/S404 was dramatically reduced compared with treatment with vehicle, while phosphorylated S262/S356 was largely unaffected by drug treatment. (C) HeLa cells expressing wild-type V5-tau were cotransfected with constitutively active GSK3 $\beta$ or empty vector. Tau was then coimmunoprecipitated with the V5 antibody, and Hsp90 binding was assessed. Hsp90 bound more avidly to tau phosphorylated by constutively active GSK3 $\beta$ compared with cells transfected with vector alone.

(MARK2/PAR-1 in Drosophila) at S262/S356 was protected from Hsp90 inhibitor-mediated degradation (16). The demonstration that CHIP regulated tau degradation by Hsp90 inhibition suggests that phosphorylation at the S262/S356 site could prevent CHIP ubiquitination. In order to address this question, transfection studies using constructs expressing either wild-type tau or a double serine-to-alanine mutant tau at 262/356 were performed to assess ubiquitination and degradation by CHIP or Hsp90 inhibition. CHIP binding and ubiquitination were completely abrogated by PAR-1-mediated phosphorylation of tau at S262/S356. Moreover, S262A/S356A tau mutants had reduced CHIP binding and no polyubiquitination similar to that of PAR-1 overexpression, suggesting that alterations in these residues, regardless of polarity, prevent CHIP ubiquitination and binding (Figure 7A).

In order to determine the impact of phosphorylation at the S262/S356 sites on Hsp90 inhibitor-mediated p-tau degradation, tau was overexpressed with either a glycogen synthase kinase $3 \beta$ (GSK3 $\beta$ ) construct encoding a primary proline-directed serine/threonine tau kinase or a PAR-1 construct in HeLa cells overexpressing V5-tau. These cells were then treated with EC102 or vehicle. Despite GSK3 $\beta$-induced increases in tau phosphorylation at S396/S404, EC102 still reduced tau levels (Figure 7B). Conversely, PAR-1-mediated phosphorylation of tau at S262/S356 prevented degradation despite EC102 treatment and resulted in increased total tau. Drug efficacy was confirmed by Hsp70 induction. These findings further suggest the necessity of functional CHIP in the mechanism of Hsp90 inhibitor-mediated p-tau degradation. Finally, coimmunoprecipitation of V5-tau showed that overexpression of GSK3 $\beta$ enhanced Hsp90 binding to tau, further demonstrating that when compared with normal tau, p-tau is a preferred client of Hsp90 (Figure 7C).

Peripheral administration of an $\mathrm{Hsp} 90$ inhibitor reduces aberrant p-tau species. In order to characterize the efficacy of EC102 in vivo, we used i.p. injections of EC102 into 2 groups of nontransgenic mice at $200 \mathrm{mg} / \mathrm{kg}$ per day for 7 days. Animals were sacrificed and their brain tissue was harvested 1 and 24 hours after the final injection. Hsp70 levels were significantly elevated in all EC102-treated mice at 1 hour after injection and persisted for 24 hours (Figure 8, A and B). In contrast, suppression of Hsp90 levels was only demonstrated at the 1-hour time point, with the levels recovering by 24 hours. These findings demonstrated that EC102 both activated HSF1 and promoted degradation of Hsp90 in the brain.

In order to assess the effect of EC102 inhibition of Hsp90 on p-tau accumulation, 8 age-matched (12-13 months) transgenic mice humanized for the tau gene (Htau mice; ref. 29) were treated with EC102 at 200 $\mathrm{mg} / \mathrm{kg}$ per day or vehicle for 7 days. Twenty-four hours following the last injection, all animals were sacrificed, and Western blot analysis was used to assess brain tau levels. Figure 8C shows Western blots of soluble tau species from the forebrain compartments of EC102-treated mice. EC102 treatment resulted in a reduction of aberrant $\mathrm{p}$-tau species, including a substantial decrease in tau phosphorylated at S396/S404 and S202/T205 (Figure 8, C and D). S262/S356 p-tau was also decreased, but only to the same extent as total tau levels. Abnormal tau species with molecular weights of approximately $64 \mathrm{kDa}(30$, 31 ) were decreased by nearly $50 \%$, but the normal tau species - in the 45-55 kDa range - were largely unaffected. Increased Hsp70 levels demonstrated drug efficacy in treated mice. In addition, neither Cdk5 nor Akt levels were affected by EC102 treatment. Both are recognized Hsp90 client proteins and have been implicated in tau phosphorylation $(32,33)$.

A bigh-affinity Hsp90 complex exists in affected regions of the AD brain. In order to determine whether Hsp90 from AD brain tissue has a higher binding affinity in EC102 than does control brain tissue, we performed competitive binding assays using a biotin-labeled GA (biotin-GA). Hsp90 complexes precipitated with biotin-GA from $\mathrm{AD}$ and control brains were incubated with increasing concentrations of EC102 (Figure 9). EC102 displaced biotin-GA from 

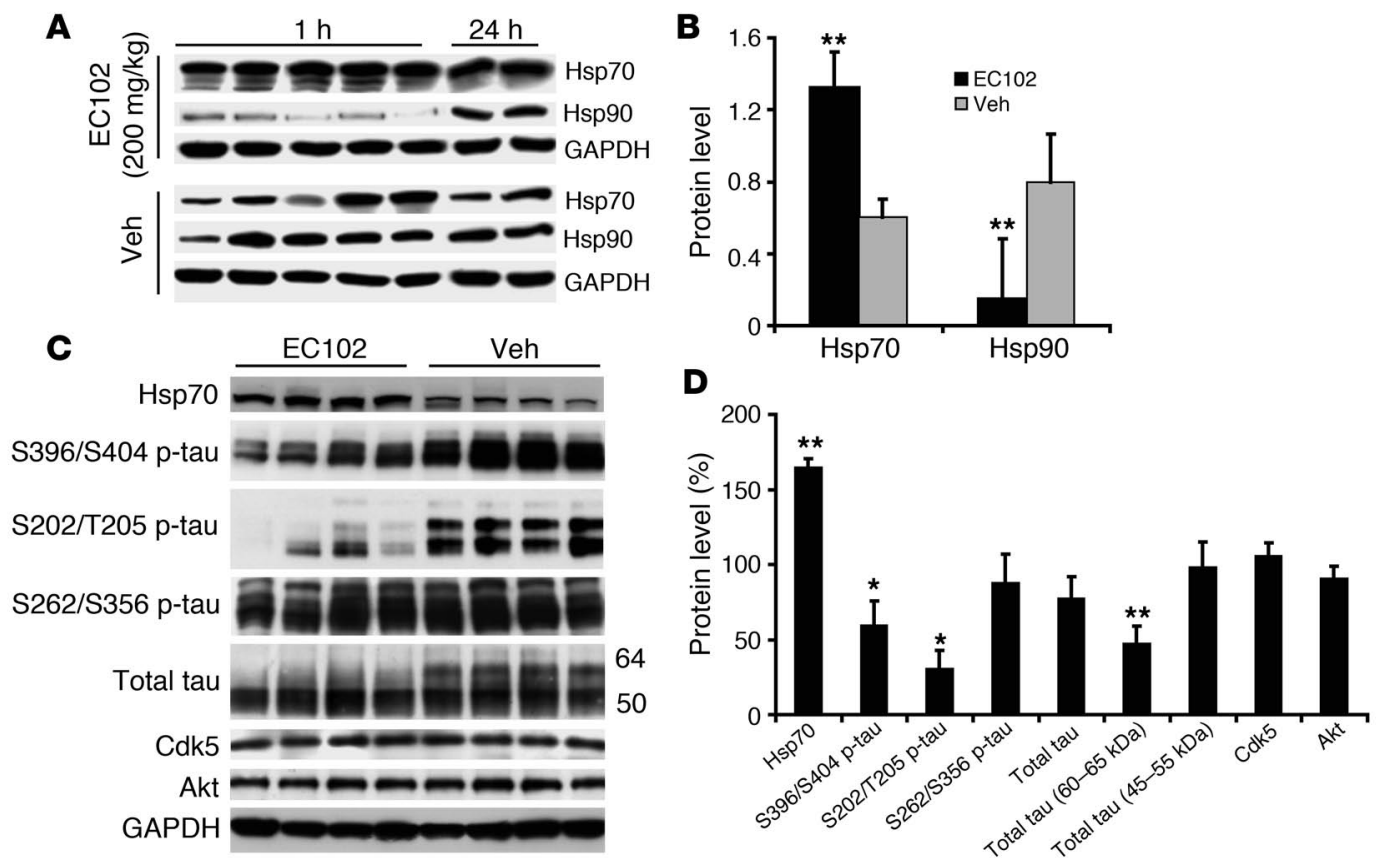

Figure 8

EC102 crosses the blood-brain barrier and acts as an Hsp90 inhibitor, decreasing p-tau levels in a mouse model of tauopathy. (A and B) Mice ( $n=7$ per group) were injected i.p. with either $200 \mathrm{mg} / \mathrm{kg}$ EC102 or equivalent vehicle once daily for 7 days. Following the final injection, 5 mice were killed within 1 hour, the 2 remaining mice were harvested 24 hours later, and their brain homogenates were assessed by Western blot (A) and those of the 5 animals harvested after 1 hour were quantitated by densitometry normalized to GAPDH (B). Hsp70 levels showed a significant increase compared with vehicle-treated mice that persisted for 24 hours. Conversely, Hsp90 levels were significantly reduced only 1 hour after EC102 administration, but were indistinguishable from vehicle-treated animals within 24 hours. Values represent optical density \pm SD. (C and D) Two cohorts of four 13- to 14-month-old Htau mice were injected i.p. with either EC102 or vehicle as above, all tissue was harvested 24 hours following the final injection, and Western blot (C) and densitometric quantitation for total tau (D) were performed. Dramatic reductions in phosphorylated S396/S404 and S202/T205 immunoreactivity in EC102-treated mice were observed. Hsp70 levels were significantly elevated in EC102-treated animals. Only the high-molecular weight species of tau (60-65 kDa), presumably p-tau species, were reduced by EC102 treatment; normal tau species $(45-55 \mathrm{kDa}$ ) remained unaffected. Levels of Hsp90 clients Cdk5 and Akt were unaltered by Hsp90 inhibition. Error bars represent SD. ${ }^{*} P<0.05 ;{ }^{*} P<0.001$ versus vehicle.

Hsp90 complexes in affected (temporal cortex) and unaffected (cerebellum) regions of $\mathrm{AD}$ brain at mean $\mathrm{IC}_{50}$ values of 6 and $6,000 \mathrm{nM}$, respectively (Figure 9). Control cases from the same areas, temporal cortex and cerebellum, had mean $\mathrm{IC}_{50}$ values of 6,000 and 7,333 nM, respectively (Figure 9). Hsp90 levels were similar among all tissues, which suggests that the observed differences were not caused by different concentrations of Hsp90 (Figure 9). Taken together, these results suggest that the Hsp90 complex in affected areas of $\mathrm{AD}$ brain, where pathologic protein accumulation is found postmortem, has a significantly higher binding affinity (approximately 1,000-fold) for EC102 than does Hsp90 derived from unaffected brain tissue of the same patients or from brain tissue of control cases. This observation is similar to that found in mutation-linked solid tumors $(15,17)$, which largely served as the basis for the introduction of Hsp90 inhibitors at the clinical level as an anticancer therapeutic.

\section{Discussion}

Recent evidence suggests that neuronal protein degradation machinery may be impaired in neurodegenerative diseases such as $\mathrm{AD}$, triggering the aberrant accumulation of tau proteins. This has been attributed, in part, to a decline in proteasomal and lysosomal function $(11,34)$. An additional potential mechanism, however, is age-associated loss of activity and expression of chaperone and associated proteins (35). The results of the present study support this hypothesis and suggest that enhancing the activity of endogenous chaperones through Hsp90 inhibition facilitates reductions in p-tau accumulation and selectively targets aberrant p-tau species that are associated with neurotoxicity. These data suggest that the surveillance mechanism of the chaperone network is highly sensitive and tightly regulated, rather than simply an unregulated "garbage disposal" for aberrant or unfolded protein species $(36,37)$. In addition, the results of these studies suggest that the administration of Hsp90 inhibitors may provide a useful clinical approach to the treatment of AD and other tauopathies.

The dynamic and conserved chaperone complex (Figure 2) that facilitates the removal of abnormal proteins such as p-tau typically works in concert with various interchangeable components (i.e., E3 ubiquitin ligases, prolyl isomerases, and other cochaperones). This process culminates either in the partial or complete refolding of the substrate or in its proteasomal degradation. Our results indicate that p-tau is a client of Hsp90 and its chaperone network. Constitutive levels of Hsp90 were required for p-tau degradation (Figure 4A). In addition, abnormal tau (hyperphosphorylated) facilitated binding to Hsp90 (Figure 7C). We have previously shown that phosphorylation of tau is constitutive, and neuronal processing of the 


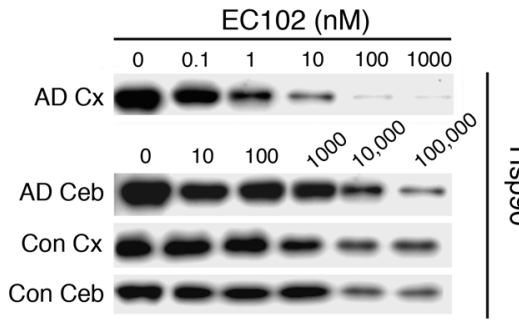

Figure 9

Hsp90 expressed in affected tissue of AD brains has significantly increased binding affinity for EC102. Brain homogenates from affected (temporal cortex, Cx) or unaffected (cerebellar cortex, $\mathrm{Ceb}$ ) areas of $3 \mathrm{AD}$ patients' brains and homogenates from the same areas in brains of 3 control cases were evaluated for binding affinity to $\mathrm{Hsp90}$ inhibitors in a competitive binding assay using a biotin-GA probe and increasing concentrations of EC102. Hsp90 derived from the temporal cortices of each $A D$ patient showed 1,000-fold greater binding affinity for EC102, with an $\mathrm{IC}_{50}$ of $6 \pm 3.6 \mathrm{nM}$, compared with Hsp90 from the cerebella, which had an $\mathrm{IC}_{50}$ of $6,000 \pm 1,000 \mathrm{nM}$ $(P<0.01)$. Controls had an $\mathrm{IC}_{50}$ of $6,000 \pm 2,000 \mathrm{nM}$ and $7,333 \pm 2,081 \mathrm{nM}$ in the temporal and cerebellar cortices, respectively. An example of the Hsp90 levels from a case and control from each area examined following competition assay between biotin-GA and EC102. Note the similar levels of Hsp90 present at $0 \mu \mathrm{M}$ EC102 among all tissues examined.

aberrant protein is a continuous process (12). Taken together, these results suggest that subtle impairment in the chaperone system that may occur with aging, coupled with even minor overproduction of abnormal client proteins, may result in aberrant accumulation and aggregation client proteins such as p-tau.

Our results demonstrate that the degradation of $\mathrm{p}$-tau follows a processing sequence through the chaperone cycle depicted in Figure 2. CHIP plays a central role in this succession of events: it has been shown to degrade Hsp70 client proteins as well as Hsp70 itself, thus acting as both a ubiquitin ligase and a stress sensor (19). CHIP also positively regulates HSF1 activity (38). In the present study, CHIP was shown to be a central regulator of constitutive and inducible chaperones. Deletion of CHIP in vivo reduced constitutive expression of the Hop chaperone (Figure 6B), an essential component for transferring proteins from the Hsp70 machine to the Hsp90 complex (23). Postdevelopmental alterations of a single chaperone could interrupt renaturation of a client protein and subsequently lead to neurotoxic protein accumulation. Our results suggest that CHIP plays a central role in mediating proper protein triage decisions, given its vast regulatory role through protein interaction and ubiquitination mechanisms.

Cellular processing of aberrant proteins includes repair, degradation, or both. The selected pathway may be lineage specific. For example, in nondividing neurons, in which apoptosis is not a viable option, the chosen protein processing pathway must be stable and sustainable for decades. Combinations of repair and degradation may also be used. Our present results and others' indicate that Hsp90 serves a somewhat passive scaffolding function, allowing other cochaperones (including P23 and Pin1) to interact with damaged client proteins. P23 maintains mature Hsp90/client complexes in a partially active state and can ultimately lead to complete refolding of the client protein $(25,39)$. Pin 1 is a tau-specific prolyl isomerase involved in tau dephosphorylation (24). When these cochaperones are individually suppressed, they promote $\mathrm{p}$-tau degradation (Figure 5). These results provide the first evidence to our knowledge that proteins involved in the refolding of abnormal Hsp90 substrates may compete with chaperones involved in degradation. In addition, Hsp90 inhibitors and both P23 and Pin 1 siRNAs promote a similar fate for client proteins in that they subvert attempts to refold proteins, thereby favoring degradation. This work suggests that the chaperone/client protein complex initially attempts to restore protein function, but should that fail, the system resorts to a protein degradation pathway.

It was previously demonstrated that CHIP antagonizes the interaction of P23 with Hsp90 (40). The present results suggest that by removing P23, CHIP is able to freely and noncompetitively bind Hsp90, thus facilitating degradation. In addition, Pin $1^{-/-}$mice demonstrate pathological tau accumulation (24), suggesting that without Pin 1 and its associated dephosphorylation and refolding, the degradation machinery may become overburdened, leading to accumulation of tau. These findings provide evidence for new substrates potentially involved in tau biology and imply a bifurcation of the chaperone network.

These previous findings, as well as our present results, demonstrate how the cellular chaperone machinery may mediate $\mathrm{p}$-tau degradation; however, it remains unclear how the chaperone complex is able to distinguish between aberrant and normal p-tau species. Tau is normally a natively unfolded protein, yet early in the pathogenesis of AD, the microtubule-binding domain (MTBD) and the $\mathrm{N}$ terminus of tau interact to form a hairpin motif recognized by the Alz-50 and MC-1 antibodies (41). This serves as a tau misfolding event and is thought to be initiated by phosphorylation (42). We previously reported that CHIP binds and ubiquitinates tau within the MTBD (11). Phosphorylation of tau by MARK2/ PAR-1 occurs at the KXGS motifs (S262 and S356). Both sites are also within the MTBD (16). In addition, p-tau from AD brains was recently shown to be polyubiquitinated at lysine residues that lie in close proximity to the MARK2/PAR-1 consensus sites (43).

The present study demonstrates (Figure 7) that CHIP is unable to bind or ubiquitinate tau that is phosphorylated or mutated at these KXGS residues, regardless of polarity (phosphorylated serine being hydrophilic, while alanine is hydrophobic). Therefore, KXGS may be a recognition consensus for both MARK2/ PAR-1 and CHIP, which would result in potential competitive binding. On the other hand, recent data suggest that nitration of N-terminal tyrosines promotes aggregation and misfolding of tau protein (44). Tau folding in AD may normally occur by a sterically favorable interaction of the nitrated tyrosine with the -OH group of serine residues within the KXGS motif. Disruption of these serines could therefore prevent misfolding and consequently also block chaperone/CHIP binding and activity. Regardless, tau phosphorylated by MARK2 has an extended half-life (45, 46), perhaps due to inhibition of degradation mechanisms. This species may thus serve as a catalyst for subsequent pathogenic events. This could suggest an antagonistic relationship between CHIP and MARK2 with regard to tau turnover. 
Table 1

siRNA sequences used in the present study

$\begin{array}{lc}\text { Gene name } & \text { Common name } \\ \text { HSP90AA1/HSPCA } & \text { Hsp90 } \\ \text { HSF1 } & \text { HSF1 } \\ \text { HSPA1A } & \text { Hsp70 } \\ \text { DNAJB1 } & \text { Hsp40 } \\ \text { STIP1 } & \text { Hop } \\ \text { STUB1 } & \text { CHIP } \\ \text { TEBP } & \text { P23 } \\ \text { PIN1 } & \text { Pin1 } \\ \text { Nonsilencing control } & -\end{array}$

ASequences for Pin1 were validated by Qiagen; the catalog number is provided in lieu of the sequence, which was not disclosed at the time of purchase.

Our results demonstrated that Hsp90 inhibition did not affect degradation of S262/S356 p-tau. These results suggest that (a) CHIP is intimately linked to tau degradation following Hsp90 inhibition and that (b) the involvement of CHIP in this process makes the pharmacologic inhibition of Hsp90 (with drugs such as EC102) extremely specific for promoting degradation of only aberrant p-tau species, leaving normal p-tau species unaffected. This is supported further by a similar pattern of specificity of the chaperones facilitating Hsp90 inhibitor-mediated degradation for mutant huntingtin protein in Huntington's disease (47) and the in vivo efficacy of Hsp90 inhibitors in a polyglutamine expansion disorder with mutant androgen receptors (48).

In the present study, we extended these observations and showed that following systemic administration, a novel Hsp90 inhibitor (EC102) can cross the blood-brain barrier and rapidly reduce levels of soluble p-tau species $(16,30)$. The Htau mouse model we used produces all 6 splice variants of the human tau protein (29), represents a rodent model for the study of the progressive pathogenesis of tauopathies such as $\mathrm{AD}$, and may serve as a reasonable in vivo model for testing pharmacologic inhibitors of soluble p-tau accumulation.

Our results demonstrated rapid and selective degradation of aberrant p-tau in the brains of Htau mice following administration of EC102 (Figure 8). Neither normal tau species nor other Hsp90 client proteins (Cdk5 or Akt) were affected by the drug. In addition, these results suggest that an intermittent dosing scheme may be possible, but exact treatment regimens remain to be determined. The potential utility of EC102 is further supported by the results of binding affinity studies in human $\mathrm{AD}$ brain tissue, in which EC102 demonstrated 1,000-fold greater affinity for Hsp90 from affected areas (temporal cortex) than from unaffected areas (cerebellar cortex) and controls from the same areas. Thus, the higher affinity present in $\mathrm{AD}$-affected brains is a result of disease (abnormal protein accumulation) rather than an intrinsic difference between brain regions. Our results demonstrate a potential for localization of therapeutic agents to areas of disease and suggest that clinically, lower concentrations of Hsp90 inhibitors could be used to treat $\mathrm{AD}$, potentially limiting unwanted effects on Hsp90/client complexes in normal tissues. Finally, the presence of Hsp90 complexes with high affinity for EC102 in other neurodegenerative disorders may justify the use of Hsp90 inhibitor therapy in other diseases.

In summary, this work demonstrates that CHIP and the Hsp90 chaperone system are essential for the proper degradation of $\mathrm{p}$-tau,
Target sequence 2 TCCCGACGATATTACTAATGA CAGGTTGTTCATAGTCAGAAT TCGAGTTTCCGGCGTCCGGAA ACCCGTCGTATTCAAAGATGT ATGACTTACATTACCAATCAA TGCCGCCACTATCTGTGTAAT CACTGTATTGATCCAAATGAT Si02662667A a protein species that undergoes continuous turnover in the neuron (12). Minor perturbations in the chaperone system with aging may retard turnover of tau, leading to aggregation and subsequent neurodegeneration. The demonstration that Hsp90 inhibitors enhance Hsp90/CHIPmediated $\mathrm{p}$-tau degradation helps to clarify the mechanisms of tau metabolism and provides a possible therapeutic strategy for the clinical management of tauopathies.

\section{Methods}

Plasmids. PAR-1 was provided by B.-W. Lu, School of Medicine, Stanford University, California, USA. Constitutively active GSK3 $\beta$ was provided by B. Wolozin, Boston University Medical Campus, Boston, Massachusetts, USA. Myc-CHIP, V5-tau, and HA-ubiquitin were generated by our lab. V5-tau S262A/S356A mutants were generated by our lab using the QuickChange Mutagenesis kit (Stratagene) following the manufacturer's protocol.

Antibodies, siRNAs, and chemicals. We obtained 12E8 (anti-S262/S356 p-tau) from P. Seubert, Elan Pharmaceuticals, San Francisco, California, USA. PHF1 (anti-S396/S404 p-tau) and CP13 (S202/T205 p-tau) were provided by P. Davies, Albert Einstein College of Medicine, Yeshiva University, New York, New York, USA. Tau 5 (anti-total tau) was provided by L. Binder, Northwestern University Medical School, Chicago, Illinois, USA. JJ3 (anti-P23) and F5 (anti-Hop) were provided by D.O. Toft, Mayo Clinic, Rochester, Minnesota, USA. Anti-V5, anti-myc, and anti-HA were obtained from Invitrogen. Anti-Hsp70, anti-Hsp40, anti-BAG-1, anti-Hsp90 $\alpha$, and anti-HSF1 were obtained from Stressgen Biotechnologies. Anti-GAPDH was obtained from BIODESIGN International. Anti-Hsc90 was obtained from BD Biosciences. Anti-Cdk5 was obtained from Santa Cruz Biotechnology Inc. Anti- $\alpha$-synuclein was obtained from Cell Signaling Technology. Anti-CHIP was generated by our group. Secondary antibodies were obtained from Southern Biotechnology Associates and Jackson Immunochemicals. All antibodies were used at a 1:1,000 dilution with the exception of PHF1 and CP13, which were used at a dilution of 1:100. All siRNAs were obtained from QIAGEN, and their sequences are listed in Table 1. siRNA efficiency for protein knockdown was validated by Western blot (Figure 3A). EC102 was generated by Conforma Therapeutics.

Cell culture and transfections. HeLa and HEK293 cells were grown in OptiMem plus 10\% FBS and passaged every 3-5 days based on $90 \%$ confluence. siRNA experiments were carried out in 6-well plates using human gene-specific validated and genome-wide siRNAs. Final siRNA concentration per well was $20 \mathrm{nM}$ in Opti-Mem, with $2 \mu \mathrm{l}$ of siLentFect transfection reagent (Bio-Rad) used per well. This mixture was incubated in a final volume of $500 \mu \mathrm{l}$ for 20 minutes and then added to $40 \%-50 \%$ confluent HeLa cells in 6-well dishes plated the previous day for a final in-well volume of $2.5 \mathrm{ml}$. Forty-eight hours after transfection, complete medium was removed and replaced with Opti-Mem (without serum) for subsequent plasmid transfection and drug treatment. For plasmid transfections, $1 \mu \mathrm{g}$ V5-tau or V5-tagged $\alpha$-synuclein was combined with Lipofectamine 2000 reagent for 15 minutes in $500 \mu \mathrm{l} \mathrm{Opti-Mem}$, and this mixture was added to the siRNA-transfected cells for 4 hours. The transfection mixture was then replaced with fresh complete media, and EC102 was added to the cells for 18-24 hours at a final concentration of $1 \mu \mathrm{M}$. For coimmunoprecipitation studies, HEK293 and HeLa cells were treated as previously described (11). Briefly, $90 \%$ confluent cells in $10-\mathrm{cm}$ dishes were transfected using 


\section{Table 2}

Characteristics of human brains used for the Hsp90 inhibitor binding study

\begin{tabular}{lccc} 
Braak NFT stage & Age (yr) & Sex & PMI (h) \\
AD & & & \\
VI & 83 & M & 3 \\
V & 83 & M & 5 \\
VI & 78 & F & 6 \\
Control & & & \\
II & 78 & F & 7 \\
III & 91 & F & 5 \\
III & 82 & M & 7 \\
\hline
\end{tabular}

Control patients (with vascular dementia) had minimal Alzheimer-type pathology, including no or sparse senile plaques and negligible neurofibrillary tangles (NFTs). AD cases and controls were matched for age, sex, brain weight, and postmortem delay. The AD group had significantly higher Braak NFT stage than did the controls, as expected.

Lipofectamine 2000 with combinations of HA-ubiquitin, wild-type V5-tau, serine-to-alanine mutant tau, myc-CHIP, PAR-1, and GSK3 $\beta$ for 4 hours and then maintained in complete media for 48 hours.

In-cell Western assay. In-cell Western analysis of p-tau levels was performed as previously described (16). Briefly, HeLa cells overexpressing V5-tau were directly fixed with formaldehyde in 96-well plates following 72 hours of growth. Plates were blocked and incubated with either (a) total tau (diluted 1:500; Dako) or GAPDH antibodies (diluted 1:1,000; Biodesign or Biosource) or (b) PHF1 (diluted 1:50; provided by P. Davies) and GAPDH antibodies overnight. Plates were washed and incubated with appropriate species-specific secondary antibodies labeled for dual near-infrared fluorescence at 700 and $800 \mathrm{~nm}$ (diluted 1:500; Rockland Immunochemicals and Invitrogen). After washing, plates were scanned using the Odyssey scanner, and relative fluorescent units were quantified. Background fluorescence was subtracted, and values for tau were normalized to GAPDH levels.

Coimmunoprecipitation with V5 tau and Hsc90/Hsp90 monoclonal antibodies. Coimmunoprecipitation to assess tau ubiquitination levels in HeLa and HEK293 cells was performed as previously described $(11,49)$. Briefly, cell supernatants following preclearing with protein $\mathrm{G}$ were incubated with anti-V5 antibody or anti-Hsc90/Hsp90 antibody and $50 \mu \mathrm{l}$ protein $\mathrm{G}$ overnight at $4^{\circ} \mathrm{C}$. Supernatants were washed 3 times in coimmunoprecipitation buffer, resuspended in sample buffer, and subjected to Western blot analyses following SDS-PAGE electrophoresis.

Rodent busbandry, treatment strategy, and tissue preparation. Htau mice (STOCK Mapt ${ }^{\text {tm1(EGFP)Klt }} \mathrm{Tg}(\mathrm{MAPT}) 8 \mathrm{cPdav} / \mathrm{J}$ ) were obtained from The Jackson Laboratory and generated as previously described (29). Briefly, these mice are transgenic for the entire human tau locus using a PAC strategy, while being null for murine microtubule associated protein tan by enhanced GFP (EGFP) insertion into exon 1 . This line was preserved on a mixed 129S4/SvJae $\times$ Swiss Webster $\times($ C57BL/6 $\times$ DBA $)$ F1 background, and genotype was assessed by PCR from tail clip digestions (29). All procedures involving transgenic mice, including treatment with Hsp90 inhibitors, were approved by the Mayo Clinic College of Medicine. An age-matched cohort (15-16 months) consisting of 8 Htau mice was injected i.p. with either a $200 \mathrm{mg} / \mathrm{kg}$ dose of EC102 or vehicle once daily for a single week and humanely euthanized following the seventh injection. For pharmacokinetic studies, EC102 was injected i.p. at doses of 200 and $400 \mathrm{mg} / \mathrm{kg}$ into 8 groups of 3 Swiss outbred CD-1 mice (Charles River Laboratories). The animals were anesthetized with isoflurane and perfused with $10 \mathrm{ml}$ sterile saline in the left ventricle. Brain tissues were collected at the indi- cated time points (Figure 1A) and flash frozen in liquid nitrogen. The brain tissues were homogenized in methanol/acetonitrile (3:2 ratio), and the homogenates were centrifuged and analyzed, after standard extraction procedures, using a Hewlett-Packard HP 1100 Series HPLC equipped with autosampler, DAD detector, and HP ChemStation software (version 2.0). A similar dosing strategy was employed for studies involving 3-month-old nontransgenic C57BL/6 mice, except that a cohort of 10 mice (5 receiving EC102 versus 5 receiving vehicle) were euthanized 1 hour following the final injection to capture reductions in Hsp90 levels based on our pharmacokinetic data. The brains were frozen on dry ice for subsequent biochemical analyses. $\mathrm{CHIP}^{-/-}$mice were generated as previously described (38). $\mathrm{CHIP}^{-/-}$and $\mathrm{CHIP}^{+/+}$mice were humanely euthanized at P30, and their brains were quickly removed for dissection and frozen on dry ice for subsequent biochemical analyses. Brain tissue was homogenized in buffer containing $50 \mathrm{mM}$ Tris, $274 \mathrm{mM} \mathrm{NaCl}, 5 \mathrm{mM} \mathrm{KCl}, 100 \mathrm{mM}$ PMSF, and a protease and phosphatase inhibitor cocktail ( $\mathrm{pH}$ 8.0). Homogenates were centrifuged at $15,400 \mathrm{~g}$ for 15 minutes, and supernatants were collected.

Western blotting. Cells from siRNA transfection studies were harvested for subsequent Western blot analysis in lysis buffer containing $50 \mathrm{mM}$ Tris$\mathrm{HCl} \mathrm{pH} 7.4,1 \mathrm{M} \mathrm{NaCl}, 0.1 \%$ Triton-X, 5 mM EDTA plus 1\% SDS, PMSF, and both a protease and phosphatase inhibitor cocktail followed by sonication. Tissue samples were homogenized and processed as described above. Protein concentrations were measured by a standard BCA assay (Pierce Biotechnology). Cell lysate samples and tissue homogenates were then heated in Laemmli's buffer, and equal amounts of protein were loaded into 10- or 15-well 10\% Tris-Glycine gels (Novex). Following transfer, blots were blocked with Blotto ( $5 \%$ non-fat dry milk in TBS plus $0.1 \%$ Triton-X 100) for 2 hours, then antibodies were applied at appropriate dilutions in Blotto overnight at $4^{\circ} \mathrm{C}$. Membranes were washed 3 times for 10 minutes in Tris-buffered saline +0.1 tritonX-100 and incubated with secondary antibody for 1 hour. Membranes were then washed 3 times for 10 minutes, and protein expression was visualized by ECL treatment and exposure to film. Bands were quantified using Scion Image by analyzing pixel density. Semiquantitative analysis was performed by densitometry, correcting protein levels for GAPDH. In order to more quantitatively assess the effect of EC102 and siRNAs on p-tau levels, the Odyssey scanner was used again for Western blot, as previously described for total tau (12). For optimal quantitative interpretation, each membrane can only be scanned by this method on the first pass. Therefore, PHF1 and GAPDH were used on the same blot in Figures 4 and 6. Quantitation was performed using Scan Image software (version Beta $3 \mathrm{~b}$ ), and PHF1 immunoreactivity was normalized to GAPDH immunoreactivity to control for loading discrepancies.

Hsp90 binding assay. Hsp90 binding assays were performed as previously described $(15,50)$. Brain homogenates from $3 \mathrm{AD}$ cases and control subjects (obtained from Mayo Clinic Jacksonville Brain Bank; Table 2) from affected (temporal cortex) and unaffected (cerebellar cortex) areas were prepared by dounce homogenization in lysis buffer (20 mM HEPES, pH 7.3, $1 \mathrm{mM}$ EDTA, $5 \mathrm{mM} \mathrm{MgCl}_{2}$, and $100 \mathrm{mM} \mathrm{KCl}$ ), incubating with or without EC102 at $0.1,1,10,100$, and $1,000 \mathrm{nM}$ for 30 minutes at $4^{\circ} \mathrm{C}$, and then incubating with biotin-GA linked to BioMag streptavidin magnetic beads (QIAGEN) for 1 hour at $4^{\circ} \mathrm{C}$. Tubes were placed on a magnetic rack, and the unbound supernatant was removed. The magnetic beads were washed 3 times in lysis buffer and heated for 5 minutes at $95.8^{\circ} \mathrm{C}$ in SDS-PAGE sample buffer. Samples were analyzed on SDS protein gels, and Western blots were performed. Bands in the Western blots were quantified using the Bio-Rad Fluor-S Multilmager, and the percentage inhibition of binding of Hsp90 to biotin-GA was calculated. The $\mathrm{IC}_{50}$ reported is the concentration of EC102 needed to cause half-maximal inhibition of binding.

Statistics. Significance was determined using a 2-tailed Student's $t$ test. ${ }^{*} P<0.05,{ }^{*} P<0.01$, and ${ }^{*}{ }^{*} P<0.001$ were assessed. 


\section{Acknowledgments}

We would like to thank D.O. Toft for his provision of antibodies against Hop and P23 as well as for his invaluable insights in interpretation of our results with respect to mechanisms of chaperone networks. We would also like to thank B.-W. Lu, B. Wolozin, L. Binder, P. Davies, and P. Seubert for providing antibodies, plasmids, and Htau mice. This work was supported by NIH grants P50-NS40256 and P01-AG17216 and by the Institute for Study of Aging.

1. Hutton, M., et al. 1998. Association of missense and $5^{\prime}$-splice-site mutations in tau with the inherited dementia FTDP-17. Nature. 393:702-705.

2. Feany, M.B., and Dickson, D.W. 1996. Neurodegenerative disorders with extensive tau pathology: a comparative study and review. Ann. Neurol. 40:139-148.

3. Gomez-Isla, T., et al. 1997. Neuronal loss correlates with but exceeds neurofibrillary tangles in Alzheimer's disease. Ann. Neurol. 41:17-24.

4. Braak, H., and Braak, E. 1991. Neuropathological staging of Alzheimer-related changes. Acta. Neuropathol. (Berl.). 82:239-259.

5. Katsuno, M., et al. 2005. Pharmacological induction of heat-shock proteins alleviates polyglutamine-mediated motor neuron disease. Proc. Natl. Acad. Sci. U. S. A. 102:16801-16806.

6. Waza, M., et al. 2006. Modulation of Hsp90 function in neurodegenerative disorders: a moleculartargeted therapy against disease-causing protein. J. Mol. Med. 84:635-646.

7. Sahara, N., et al. 2005. In vivo evidence of CHIP up-regulation attenuating tau aggregation. J. Neurochem. 94:1254-1263.

8. Hamos, J.E., et al. 1991. Expression of heat shock proteins in Alzheimer's disease. Neurology. 41:345-350.

9. Perez, N., et al. 1991. Increased synthesis and accumulation of heat shock 70 proteins in Alzheimer's disease. Brain Res. Mol. Brain Res. 11:249-254.

10. Shimura, H., Miura-Shimura, Y., and Kosik, K.S. 2004. Binding of tau to heat shock protein 27 leads to decreased concentration of hyperphosphorylated tau and enhanced cell survival. J. Biol. Chem. 279:17957-17962.

11. Petrucelli, L., et al. 2004. CHIP and Hsp70 regulate tau ubiquitination, degradation and aggregation. Hum. Mol. Genet. 13:703-714.

12. Dickey, C.A., et al. 2006. Deletion of the ubiquitin ligase CHIP leads to the accumulation, but not the aggregation, of both endogenous phosphoand caspase-3-cleaved tau species. J. Neurosci. 26:6985-6996

13. Panaretou, B., et al. 1998. ATP binding and hydrolysis are essential to the function of the Hsp90 molecular chaperone in vivo. EMBO J. 17:4829-4836.

14. Ciechanover, A., and Brundin, P. 2003. The ubiquitin proteasome system in neurodegenerative diseases: sometimes the chicken, sometimes the egg. Neuron. 40:427-446.

15. Kamal, A., et al. 2003. A high-affinity conformation of Hsp90 confers tumour selectivity on Hsp90 inhibitors. Nature. 425:407-410.

16. Dickey, C.A., et al. 2006. HSP induction mediates selective clearance of tau phosphorylated at proline-directed Ser/Thr sites but not KXGS (MARK) sites. FASEB J. 20:753-755.

17. Biamonte, M.A., et al. 2006. Orally active purinebased inhibitors of the heat shock protein 90 . J. Med. Chem. 49:817-828.

Received for publication July 16, 2006, and accepted in revised form December 19, 2006.

Address correspondence to: Leonard Petrucelli, Mayo Clinic, 4500 San Pablo Road, Jacksonville, Florida 32224, USA. Phone: (904) 9532855; Fax: (904) 953-7370; E-mail: petrucelli.leonard@mayo.edu.

Chad Dickey's present address is: University of South Florida College of Medicine, Tampa, Florida, USA.

18. Dickey, C.A., et al. 2005. Development of a high throughput drug screening assay for the detection of changes in tau levels - proof of concept with HSP90 inhibitors. Curr. Alzheimer Res. 2:231-238.

19. Qian, S.B., McDonough, H., Boellmann, F., Cyr, D.M., and Patterson, C. 2006. CHIP-mediated stress recovery by sequential ubiquitination of substrates and Hsp70. Nature. 440:551-555.

20. Chang, H.C., Nathan, D.F., and Lindquist, S. 1997. In vivo analysis of the Hsp90 cochaperone Sti1 (p60). Mol. Cell. Biol. 17:318-325.

21. Frydman, J., and Hohfeld, J. 1997. Chaperones get in touch: the Hip-Hop connection. Trends Biochem. Sci. 22:87-92.

22. Chen, S., and Smith, D.F. 1998. Hop as an adaptor in the heat shock protein 70 (Hsp70) and hsp90 chaperone machinery. J. Biol. Chem. 273:35194-35200.

23. Johnson, B.D., Schumacher, R.J., Ross, E.D., and Toft, D.O. 1998. Hop modulates Hsp70/Hsp90 interactions in protein folding. J. Biol. Chem. 273:3679-3686

24. Liou, Y.C., et al. 2003. Role of the prolyl isomerase Pin 1 in protecting against age-dependent neurodegeneration. Nature. 424:556-561.

25. Grenert, J.P., et al. 1997. The amino-terminal domain of heat shock protein 90 (hsp90) that binds geldanamycin is an ATP/ADP switch domain that regulates hsp90 conformation. J. Biol. Chem. 272:23843-23850.

26. Li, T., et al. 2004. Identification of the gene for vitamin K epoxide reductase. Nature. 427:541-544.

27. Soifer, H.S., Zaragoza, A., Peyvan, M., Behlke, M.A., and Rossi, J.J. 2005. A potential role for RNA interference in controlling the activity of the human LINE-1 retrotransposon. Nucleic Acids Res. 33:846-856.

28. Grelle, G., et al. 2006. Identification of VCP/p97, carboxyl terminus of Hsp70-interacting protein (CHIP), and amphiphysin II interaction partners using membrane-based human proteome arrays. Mol. Cell. Proteomics. 5:234-244.

29. Andorfer, C., et al. 2003. Hyperphosphorylation and aggregation of tau in mice expressing normal human tau isoforms. J. Neurochem. 86:582-590.

30. Santacruz, K., et al. 2005. Tau suppression in a neurodegenerative mouse model improves memory function. Science. 309:476-481.

31. Tronjanowski, J.Q., and Lee, V.M. 2005. Pathological tau: a loss of normal function or a gain in toxicity? Nat. Neurosci. 8:1136-1137.

32. Kobayashi, S., et al. 1993. A cdc2-related kinase PSSALRE/cdk5 is homologous with the $30 \mathrm{kDa}$ subunit of tau protein kinase II, a proline-directed protein kinase associated with microtubule. FEBS Lett. 335:171-175.

33. Hong, M., and Lee, V.M. 1997. Insulin and insulin-like growth factor-1 regulate tau phosphorylation in cultured human neurons. J. Biol. Chem. 272:19547-19553.

34. Barrachina, M., Maes, T., Buesa, C., and Ferrer, I. 2006. Lysosome-associated membrane protein 1
(LAMP-1) in Alzheimer's disease. Neuropathol. Appl. Neurobiol. 32:505-516

35. Cohen, E., Bieschke, J., Perciavalle, R.M., Kelly, J.W., and Dillin, A. 2006. Opposing activities protect against age-onset proteotoxicity. Science. 313:1604-1610.

36. Goldberg, A.L., Stein, R., and Adams, J. 1995. New insights into proteasome function: from archaebacteria to drug development. Chem. Biol. 2:503-508.

37. Dalton, W.S. 2004. The proteasome. Semin. Oncol. 31(Suppl. 16):3-9; discussion 33.

38. Dai, Q., et al. 2003. CHIP activates HSF1 and confers protection against apoptosis and cellular stress. EMBO J. 22:5446-5458.

39. Grenert, J.P., Johnson, B.D., and Toft, D.O. 1999. The importance of ATP binding and hydrolysis by hsp90 in formation and function of protein heterocomplexes. J. Biol. Chem. 274:17525-17533.

40. Connell, P., et al. 2001. The co-chaperone CHIP regulates protein triage decisions mediated by heat-shock proteins. Nat. Cell Biol. 3:93-96.

41. Weaver, C.L., Espinoza, M., Kress, Y., and Davies, P. 2000. Conformational change as one of the earliest alterations of tau in Alzheimer's disease. Neurobiol. Aging. 21:719-727.

42. Ghoshal, N., et al. 2001. Tau-66: evidence for a novel tau conformation in Alzheimer's disease. J. Neurochem. 77:1372-1385.

43. Cripps, D., et al. 2006. Alzheimer's disease-specific conformation of hyperphosphorylated phftau is polyubiquitinated through lys-48, lys-11, and lys- 6 ubiquitin conjugation. J. Biol. Chem. 281:10825-10838.

44. Reynolds, M.R., Berry, R.W., and Binder, L.I. 2005. Site-specific nitration differentially influences tau assembly in vitro. Biochemistry. 44:13997-14009.

45. Biernat, J., et al. 2002. Protein kinase MARK/PAR-1 is required for neurite outgrowth and establishment of neuronal polarity. Mol. Biol. Cell. 13:4013-4028.

46. Mandelkow, E.M. 2006. The modes and pathways of Tau's toxicity to neurons in neurodegeneration. Paper presented at the Keystone Symposia Conference on Alzheimer's Disease: Genes, Cellular Pathways and Therapies. February 21-26. Breckenridge, Colorado, USA

47. Sittler, A., et al. 2001. Geldanamycin activates a heat shock response and inhibits huntingtin aggregation in a cell culture model of Huntington's disease. Hum. Mol. Genet. 10:1307-1315.

48. Waza, M., et al. 2005. 17-AAG, an Hsp90 inhibitor, ameliorates polyglutamine-mediated motor neuron degeneration. Nat. Med. 11:1088-1095.

49. Petrucelli, L., and Dawson, T.M. 2004. Mechanism of neurodegenerative disease: role of the ubiquitin proteasome system. Ann. Med. 36:315-320.

50. Castro, J.E., et al. 2005. ZAP-70 is a novel conditional heat shock protein 90 (Hsp90) client: inhibition of Hsp90 leads to ZAP-70 degradation, apoptosis, and impaired signaling in chronic lymphocytic leukemia. Blood. 106:2506-2512. 